\title{
Sindrome da anticorpi antifosfolipidi primaria (PAPS): descrizione di un caso clinico evoluto in lupus eritematoso sistemico
}

\author{
Primary antiphospholipid syndrome progressing to systemic lupus \\ erythematosus: a case report
}

\author{
Rocco Manganelli *, Salvatore lannaccone, Walter De Simone
}

UO Nefrologia e Dialisi, Azienda Ospedaliera di Rilievo Nazionale e di Alta Specialità "S. Giuseppe Moscati", Avellino

Ricevuto il 27 marzo 2009; accettato l'11 settembre 2009

disponibile online il 20 aprile 2010

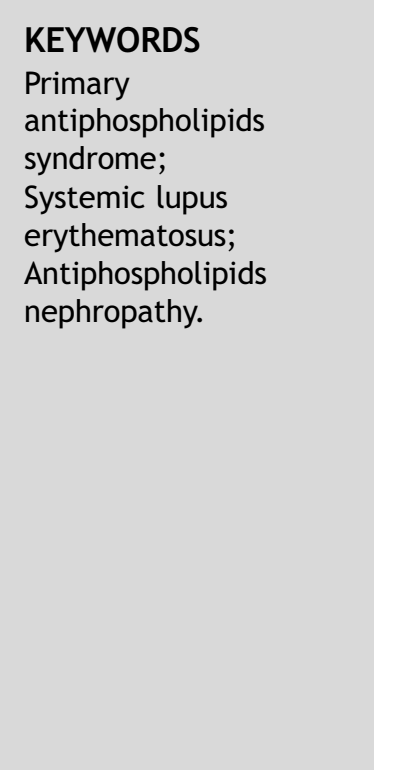

\begin{abstract}
Summary
Introduction: Primary antiphospholipid syndrome (APS) is a thrombophilic disease that should be suspected in the presence of thrombotic events associated with hematologic abnormalities such as thrombocytopenia and prolongation of the activated partial thromboplastin time. The diagnosis must be confirmed by the demonstration of autoantibodies directed against anionic phospholipids and/or phospholipid-binding proteins. The disease can cause arterial thrombosis in any vascular district, including those of the kidney and central nervous system.

Case report: In 2006 a 29-year-old male presented with kidney and brain involvement that was attributed to primary APS. The clinical diagnosis was confirmed by the results of a renal biopsy, which excluded the presence of systemic lupus erythematosus (SLE). The patient remained stable through 32 months of follow-up and then developed a malar rash with deteriorating renal function, decreasing platelet count, and reduced complement levels. Serological studies revealed positivity for ANA (homogeneous pattern), dsDNA, ACA, and beta-2-glycoprotein-1 antibodies. The diagnosis was revised to APS secondary to SLE.

Conclusions: A diagnosis of primary APS should not be considered permanent: progression to SLE can occur, in some cases years after the original diagnosis. This case highlights the importance of ongoing follow-up of patients diagnosed with primary APS to detect changes that herald the emergence of SLE.

(C) 2010 Elsevier Srl. All rights reserved.
\end{abstract}

\footnotetext{
* Corrispondenza: UO Nefrologia e Dialisi, AORN "S. Giuseppe Moscati", v.le Otranto - 83100 Avellino.

E-mail: rocco.manganelli@tin.it (R. Manganelli).
} 


\section{Presentazione del caso}

Maschio, caucasico, di anni 29, fu ricoverato nel 2006 a causa di dispnea da sforzo, accompagnata da intensa cefalea insorta 3 giorni prima. Furono riscontrate ipertensione arteriosa di III grado $(180 / 150 \mathrm{mmHg})$ e insufficienza renale a diuresi conservata (creatinina $4,5 \mathrm{mg} / \mathrm{dL}$ ). Le indagini strumentali evidenziarono severa compromissione cerebrale e cardiaca.

La TC dell'encefalo senza mezzo di contrasto descrisse multiple aree ipodense lacunari a sede occipitale sinistra, frontale destra e parietale profonda destra; la RMN cerebrale confermò l'estensione delle lesioni evidenziate dalla TC e indicò anche un parziale coinvolgimento del lobo sinistro del cervelletto, descrivendo molteplici aree di aumentata intensità del segnale compatibili con piccoli foci di ischemia subcorticale.

L'ecocardiogramma transtoracico evidenziò ipertrofia del ventricolo sinistro associata a modica dilatazione delle camere di sinistra, moderata insufficienza mitralica e deficit di pompa del ventricolo sinistro (FE 48\%).

Dopo la stabilizzazione farmacologica delle condizioni cardiocircolatorie (nitroglicerina ev, furosemide ev, betabloccanti e calcio-antagonisti), il paziente appariva in buono stato generale, asintomatico, con discreto controllo dei valori di PA (140/90 mmHg). L'obiettività, compreso l'esame neurologico, era negativa. II laboratorio evidenziava creatinina stabile $(4,5 \mathrm{mg} / \mathrm{dL})$ e valori di azotemia pari a $120 \mathrm{mg} / \mathrm{dL}$; l'esame delle urineera positivo per proteinuria, con escrezione proteica nelle 24 ore pari a $1,4 \mathrm{~g}$. All'esame emocromocitometrico risultavano emoglobina $12,2 \mathrm{~g} / \mathrm{dL}$ e trombocitopenia $\left(82.000 / \mathrm{mm}^{3}\right)$ in assenza di segni laboratoristici indicativi di emolisi microangiopatica (assenza di schizociti nello striscio periferico; aptoglobina e bilurubinemia normali). I test emocoagulativi di routine erano normali (PT 87\%, fibrinogeno $250 \mathrm{mg} / \mathrm{dL})$, ma si rilevava allungamento dell'aPTT $\left(73,80^{\prime \prime}\right)$. Quadro proteico, complementemia (C3-C4), funzionalità epatica ed elettroliti erano nella norma. Il pannello autoanticorpale evidenziava debole positività per gli ANA speckled (1:80); la ricerca per anti-dsDNA e anti-ENA era negativa, così come per gli ANCA. La sierologia per marcatori virali $\mathrm{HbsAg}, \mathrm{HCV}$ e HIV era negativa. Al riguardo degli indici infiammatori, la VES evidenziava un modico incremento $(59 \mathrm{~mm} / \mathrm{h})$ e la PCR era nella norma.

La contemporanea presenza di coinvolgimento cerebrale di tipo micro-multinfartuale, insufficienza renale, trombocitopenia e prolungamento dell'aPTT orientarono a una possibile diagnosi di sindrome da anticorpi antifosfolipidi (Antiphospholipids Syndrome, APS).

Lo screening per la ricerca del Lupus Anticoagulant (LA) rivelò la positività di tre su sei test fosfolipide-dipendenti esplorati (comprensivi di KCT, DRVVT e aPTT con fosfolipidi non vegetali), confermata dalla mancata correzione dei test coagulativi alle prove di mixing 50:50 con plasma di donatore sano, correzione invece ottenuta con l'aggiunta di fosfolipidi al mix. Gli ACA (Anticardiolipin Antibody) misurati con metodica ELISA erano intensamente positivi per l'isotipo lgG: $137 \mathrm{GPL} U(\mathrm{vn}<15 \mathrm{GPL} U)$.

In settima giornata dal ricovero il paziente fu sottoposto a biopsia renale ecoguidata per chiarire la natura e l'estensione del processo renale. L'esame del frammento per la MO (19 glomeruli) mostrò, a carico di due arteriole interlobulari,

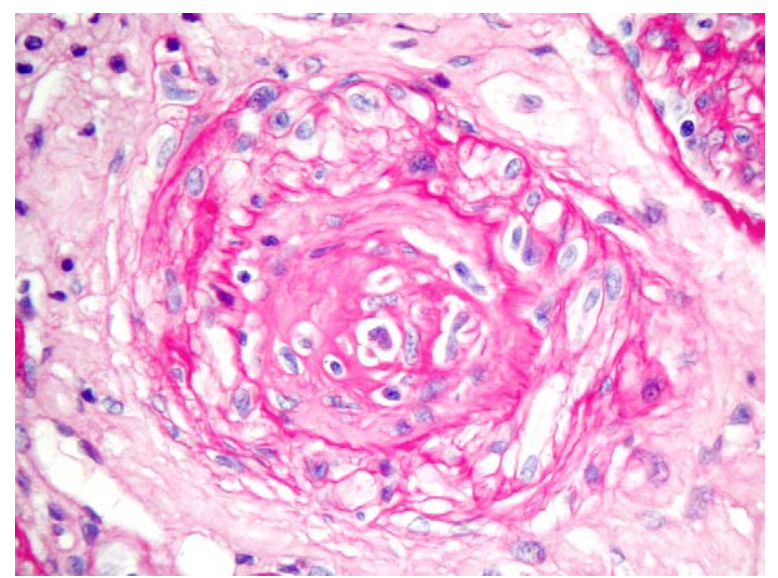

Figura 1 Occlusione trombotica completa del lume arteriolare associata a sclerosi intimale con iperplasia di cellule miofibroblastiche subendoteliali. Coesiste iperplasia delle cellule muscolari della media (PAS 250x).

sclerosi intimale e iperplasia intimale subendoteliale di cellule miofibroblastiche con coesistente iperplasia delle cellule muscolari della tonaca media. In una delle due arteriole erano presenti trombizzazione organizzata e occlusione completa del lume (Fig. 1). I glomeruli mostravano sclerosi globale in 3, nei restanti sclerosi segmentale della matrice mesangiale, con "collassamento" delle anse dei capillari glomerulari (Figg. 2-3). L'immunofluorescenza risultò negativa.

La diagnosi conclusiva fu di sindrome da anticorpi antifosfolipidi primaria (PAPS) associata a glomerulosclerosi focale e segmentaria con pattern "collapsing".

In decima giornata dall'esecuzione della biopsia renale fu iniziata terapia con warfarin allo scopo di mantenere l'INR intorno a 3.

Alle dimissioni il paziente fu monitorato ogni 3 mesi per quanto riguarda la funzione renale, ogni 6 mesi per gli autoanticorpi e il complemento e ogni mese per l'emocromo, la

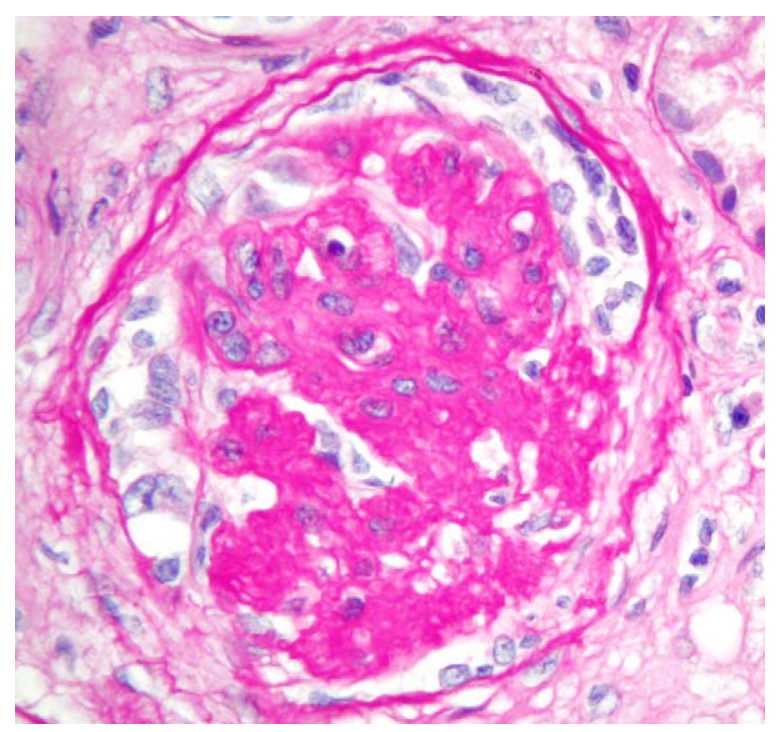

Figura 2 Lesioni sclerotiche segmentali glomerulari avanzate con ispessimento della matrice mesangiale e obliterazione dei lumi capillari (PAS 250x). 


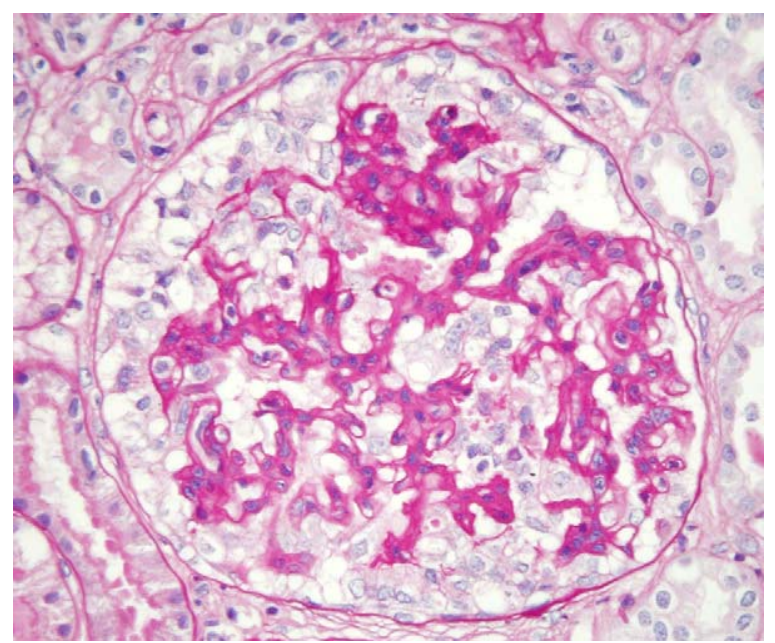

Figura 3 Aspetto "collassato" del ciuffo glomerulare. Iperplasia delle cellule podocitarie (PAS 250x).

conta piastrinica e la coagulazione, per guidare gli aggiustamenti posologici del warfarin. Il follow-up era caratterizzato da completa stabilità clinica e bioumorale. A 24 mesi i valori di creatinina erano intorno a $1,6 \mathrm{mg} / \mathrm{dL}$, la proteinuria delle 24 ore non $>1,5 \mathrm{~g}$, le piastrine $100.000 / \mathrm{mm}^{3}$ (recupero funzionale renale e risalita piastrinica erano presenti sin dal terzo mese), buon controllo dei valori di PA $(125 / 80 \mathrm{mmHg})$, esami autoanticorpali negativi.

Dopo 32 mesi cominciò ad apparire rash malare e si rilevarono incremento della creatinina $(2,5 \mathrm{mg} / \mathrm{dL})$ e dell'escrezione proteica (intorno a $2,5 \mathrm{~g} / 24$ ore) e riduzione della conta piastrinica $\left(60-70.000 / \mathrm{mm}^{3}\right)$. A 35 mesi comparve riduzione del complemento: $\mathrm{C} 30,70 \mathrm{~g} / \mathrm{L}$ (range normale: 0,90-1,80 g/L); C4 $0,07 \mathrm{~g} / \mathrm{L}$ (range normale: $0,10-0,40 \mathrm{~g} /$ L). Il work-up sierologico rivelò: pattern ANA omogeneo positivi a titolo $1: 160$, anti-dsDNA positivi (IFI Crithidia) con alto titolo (1.220 U/L; ELISA-DNA ricombinante). La ricerca di anticorpi antifosfolipidi evidenziò: ACA IgG 150 GPL U (vn < 15); ACA IgM 2 MPL U (vn < 15); anticorpi anti-beta-2-glicoproteina-1 ( $\beta 2$-GP-1) positivi.

All'esame fisico il paziente era afebbrile; PA $130 / 90 \mathrm{mmHg}$ (carvedilolo $25 \mathrm{mg}$; ramipril $5 \mathrm{mg}$; amlodipina $10 \mathrm{mg}$ ); FC 72 bpm; ritmo cardiaco regolare con lieve soffio da rigurgito mitralico; non rumori aggiunti all'esame polmonare. L'esame ispettivo rivelava rash malare con cute per il resto sana, non edema periferico, cianosi, artrite, clubbing. Ulteriori esami di laboratorio mostravano VES $80 \mathrm{~mm} / \mathrm{h}$ (vn 4-25 mm/h); PCR 8,90 (vn 0,00-7 mg/L). L'esame microscopico del sedimento urinario mostrava 20-25 globuli rossi/HPF con qualche cilindro ematico.

Fu posta diagnosi di Lupus Eritematoso Sistemico con APS secondaria (2009). I principali esami diagnostici effettuati nel 2006 e nel 2009 sono sintetizzati nelle tabelle 1 e 2 .

Fu stabilito un incontro fra il nefrologo, il reumatologo, il paziente e la moglie per discutere la prosecuzione dell'iter diagnostico e terapeutico: si decise di non eseguire, almeno al momento, una nuova biopsia renale, considerati il rischio bioptico associato alla piastrinopenia, il rischio trombotico generale dovuto alla necessità di sospendere il warfarin in vista della procedura bioptica e, non ultima, la resistenza del
Tabella 1 Principali esami indicativi della diagnosi di PAPS nel 2006.

\begin{tabular}{ll}
\hline aPTT & $73,80^{\prime \prime}$ (prolungato) \\
Trombocitopenia & $82.000 / \mathrm{mm}^{3}$ \\
ANA speckled & $1: 80$ (positivo) \\
TC-RMN encefalo & Positive \\
Lupus Anticoagulant & Positivo \\
ACA IgG & $137 \mathrm{GPL}$ U (valori \\
& normali $<15 \mathrm{GPL} U)$
\end{tabular}

Tabella 2 Principali esami indicativi della diagnosi di APS secondaria a LES nel 2009.

\begin{tabular}{ll}
\hline Anti-dsDNA & $1: 1.200$ (positivo) \\
ANA omogeneo & $1: 160$ (positivo) \\
C3 & $0,70 \mathrm{~g} / \mathrm{L}$ (valori normali $0,90-1,80 \mathrm{~g} / \mathrm{L}$ ) \\
C4 & $0,07 \mathrm{~g} / \mathrm{L}$ (valori normali $0,10-0,40 \mathrm{~g} / \mathrm{L}$ ) \\
ACA IgG & $150 \mathrm{GPL} U$ (valori normali $<15 \mathrm{GPL} \mathrm{U}$ ) \\
Anti- $\beta 2-G P-1$ & Positivi
\end{tabular}

paziente. Fu praticata terapia con boli di metilprednisolone $1 \mathrm{~g} /$ die per 3 giorni consecutivi, seguita da prednisone per os $1 \mathrm{mg} / \mathrm{kg}$ di peso corporeo. Fu iniziata immunosoppressione con micofenolato mofetil $2 \mathrm{~g} /$ die.

Gli autori dichiarano che lo studio presentato è stato realizzato in accordo con gli standard etici stabiliti nella Dichiarazione di Helsinki, e che il consenso informato è stato ottenuto da tutti i partecipanti prima del loro arruolamento allo studio.

\section{Discussione}

La sindrome da antifosfolipidi è caratterizzata da ricorrenti eventi trombotici arteriosi o venosi, aborti ricorrenti e trombocitopenia. Le tipiche alterazioni di laboratorio che consentono di porre la diagnosi comprendono la positività del test del Lupus Anticoagulant (LA) o elevati e persistenti livelli di anticorpi anticardiolipina (ACA) o elevato e persistente titolo di anticorpi anti-beta-2-glicoproteina-1 ( $\beta 2-\mathrm{GP}-1)$ [1].

La definizione "lupus anticoagulant" risale alla scoperta di Conley e Hartman, che negli anni Cinquanta evidenziarono nel siero di due pazienti affetti da LES la presenza in vitro di un anticoagulante. Ulteriori studi di laboratorio mostrarono che l'anticoagulante era un'immunoglobulina che inibiva il complesso di attivazione della protrombina. Paradossalmente il LA, mentre in vitro causava prolungamento dei test di coagulazione, in vivo era responsabile dell'effetto opposto, cioè di trombosi. All'inizio degli anni Ottanta, Harris identificò gli ACA in un subset di pazienti con LES, ma apparve subito chiaro che questi anticorpi riconoscevano non solo la cardiolipina, bensì tutta la famiglia di fosfolipidi a carica elettrica negativa [2]. Nel 1990 si scoprì che, in assenza della proteina sierica $\beta 2$-GP-1, la reazione verso i fosfolipidi in fase solida non poteva avvenire [3]. Si comprese quindi che gli anticorpi non erano diretti contro i fosfolipidi anionici (aPL), 
ma contro le proteine plasmatiche legate a superfici anioniche come i fosfolipidi.

Gli antigeni più studiati di questi autoanticorpi sono stati la $\beta 2-G P-1$ e la protrombina, sebbene nel siero dei pazienti si sia riscontrato un cospicuo numero di autoanticorpi che riconoscono altri tipi di proteine PL-binding quali le proteine $\mathrm{C}$ e S, l'annessina $\mathrm{V}$, il fattore $\mathrm{H}$ del complemento, il chininogeno ad alto e basso peso molecolare, la precallicreina, il fattore XI, il fattore VII/VIla.

Gli anticorpi anti- $\beta 2-$-GP-1 sono responsabili della reattività anticardiolipina presente nel siero dei pazienti con APS, mentre l'attività del LA è attribuibile sia agli anti- $\beta 2-G P-1$ sia ad anticorpi antiprotrombina.

La diagnosi di APS si fonda sulla valutazione di criteri clinici (eventi trombotici confermati con tecniche di imaging, doppler o istopatologiche e/o aborti ricorrenti) e laboratoristici (positività persistente degli ACA presenti a titolo medio 0 alto e/o anti- $\beta 2-G P-1$ a titolo $>99^{\circ}$ percentile e/o positività del LA ottenuto secondo metodo raccomandato dalle linee guida dell'International Society on Thrombosis and Haemostasis. La contemporanea presenza di un criterio clinico e di uno laboratoristico è sufficiente per diagnosticare la sindrome $[4,5]$.

Nel 2006 l'imaging cerebrale (TC e RMN) del nostro paziente mostrava le conseguenze ischemiche di ripetuti eventi trombotici a livello del circolo cerebrale, ed è noto che il sistema nervoso centrale è la sede più frequente di trombosi arteriose, responsabili di uno spettro clinico polimorfo e comprensivo di semplice cefalea o emicrania, disturbi neurocognitivi, manifestazioni neuropsichiatriche come depressione e psicosi, corea, convulsioni, sindrome di Guillain-Barré, attacchi ischemici transitori e stroke, demenza multinfartuale [6]. L'elevata frequenza del coinvolgimento del sistema nervoso centrale in corso di APS sembra essere in relazione con una particolare vulnerabilità dell'endotelio cerebrale all'azione degli aPL: infatti la $\beta 2$ GP-1, principale target antigenico degli aPL, sembra essere espressa in maggiore quantità e stabilmente legata alla cellule dell'endotelio cerebrale; ne deriva un particolare tropismo degli aPL per il sistema nervoso centrale, con conseguente attivazione cellulare ed espressione di un fenotipo endoteliale procoagulante e proinfiammatorio [7].

Peraltro, la cefalea persistente presentata dal nostro paziente poteva anche essere causata dagli elevati valori di pressione arteriosa (PA diastolica $150 \mathrm{mmHg}$ ), e l'ipertensione, spesso maligna, è il sintomo di coinvolgimento renale più frequente nelle serie della letteratura, assieme all'insufficienza renale e alla proteinuria di grado lieve [8]. Essa è secondaria all'intensa stimolazione dell'asse reninaangiotensina-aldosterone determinata dalle lesioni dei vasi renali [9].

Il tipo di coinvolgimento renale attribuile alla PAPS (APS Nephropathy, APSN) si fonda ancora oggi sul lavoro di Nochy et al. del 1999 [10]: lesioni vaso-occlusive dei vasi intrarenali caratterizzate dalla stretta associazione di trombosi acute e lesioni croniche arterio-arteriolari responsabili di zone di atrofia ischemica corticale. Dalla diversa prevalenza delle lesioni dipende la presentazione clinica della malattia renale.

- La prevalenza di TMA (Microangiopathy Thrombotic) è responsabile di insufficienza renale acuta, spesso associata a ipertensione maligna, proteinuria nefrosica o subnefrosica ed eccezionalmente ad anemia emolitica microangiopatica [11].

- La prevalenza di FIH (Fibrous Intimal Hyperplasia), caratterizzata da ispessimento intimale da parte di cellule miofibroblastiche che conduce nel tempo a occlusione arteriolare per effetto di proiezioni fibrose e trombi organizzati, assieme alla FCA (Focal Cortical Atrophy), caratterizzata da aree focali di fibrosi e retrazione della corteccia subcapsulare su base ischemica, si associa a ipertensione moderata, insufficienza renale cronica e proteinuria di lieve entità [12].

È altamente probabile che $i$ foci di FCA rappresentino lesioni simili o identiche alle piccole aree ischemiche descritte nell'imaging cerebrale.

In anni più recenti lo spettro delle lesioni renali si è ampliato in virtù delle frequenti segnalazioni di glomerulonefriti croniche associate a PAPS nelle quali le lesioni vascolari della APSN sono meno predominanti o addirittura assenti: nefropatia membranosa, glomerulosclerosi focale e segmentaria, nefropatia a depositi mesangiali di C3, "minimal change disease" e vasculite paucimmune [13-15].

Nel caso del nostro paziente il quadro istologico era caratterizzato dalle lesioni vascolari della APSN con coesistenza di alterazioni glomerulari riferibili a glomerulosclerosi focale e segmentaria con pattern collapsing [16].

Dal punto di vista clinico, per diagnosticare la PAPS occorre escludere malattie autoimmuni sottostanti: principalmente il LES, che si accompagna ad APS nel 37\% dei casi, mentre altre, come l'artrite reumatoide, la sclerosi sistemica progressiva e la sindrome di Sjøgren, solo nel 4\% [17]. Tuttavia, molte manifestazioni cliniche dell'APS costituiscono esse stesse criteri classificativi del LES; se si considerano i criteri dell'American College of Rheumatology (ACR) aggiornati al 1997 [18], essi includono la positività ad alto titolo degli aPL riducendo i criteri utili per la diagnosi di LES da 4/11 a 3/11. Nel 2006, la contemporanea presenza nel nostro paziente di tre criteri stabiliti dall'American Rheumatism Association (ARA) - positività per gli aPL, trombocitopenia e insufficienza renale con proteinuria $>500 \mathrm{mg} / 24$ ore - ha reso difficile la diagnosi differenziale. Per questo è necessario enfatizzare la persistente negatività di specificità anticorpali tipiche del LES (anti-ds-DNA e anti-ENA). La debole positività di anticorpi antinucleo (ANA 1:80) riscontrata nel caso in esame è considerata compatibile con diagnosi di PAPS, in quanto la frequenza degli ANA in pazienti affetti da PAPS varia dal 41 al 77\% [19,20]. Nel nostro paziente, nel 2006 l'assenza dei criteri ACR e l'istopatologia renale negativa per una glomerulonefrite da immunocomplessi confermavano la diagnosi di PAPS.

Vi era tuttavia la possibilità che il paziente fosse affetto da una sindrome lupus-like di passaggio verso il LES conclamato [21]. Nel 2009 la diagnosi di LES è stata sospettata quando ha sviluppato rash malare, peggioramento della funzione renale accompagnato a incremento della proteinuria e sedimento urinario attivo. La diagnosi è stata confermata dalla positività per gli ANA e degli anticorpi anti-dsDNA ad alto titolo. Peraltro, gli anticorpi anti-dsDNA correlano con la presenza e la severità della nefropatia lupica, così come l'ipocomplementemia nel LES è più comune in presenza di evidente nefrite: la concentrazione di $\mathrm{C} 4$ più depressa del $\mathrm{C} 3$ suggerisce l'attivazione della via classica del complemento [22]. Le manifestazioni istopatologiche della nefrite lupica 
sono raggruppate nelle sei classi stabilte dalla World Health Organization (WHO) [23]; le classi III e IV, rispettivamente glomerulonefrite focale e diffusa, sono distinte in base alla percentuale dei glomeruli coinvolti e generalmente le lesioni renali si presentano con ematuria microscopica, con o senza cilindri ematici e vari gradi di insufficienza renale. Viceversa la classe V, glomerulonefrite membranosa, manifesta tipicamente proteinuria in range nefrosico. Nel nostro paziente, la presenza di un incremento della creatinina, della microematuria con cilindri ematici e l'incremento della proteinuria a livelli non nefrosici suggeriscono molto probabilmente una classe III o IV. É necessario sottolineare che non si può porre la diagnosi di classe basandosi esclusivamente sulla presentazione clinica e, siccome il trattamento ottimale varia con il tipo di malattia glomerulare, solo la biopsia renale offre la diagnosi definitiva [24,25]. Non potendo disporre di una rebiopsia renale, nel caso in esame si è adottato uno schema di terapia di induzione valido per la nefropatia lupica severa, definendo quest'ultima in base alla presenza all'istologia di classe III severa o IV e/o sindrome nefrosica e/o ridotta funzione renale \pm ipertensione alla presentazione clinica [22].

La scelta del micofenolato mofetil si è basata sul fatto che il farmaco ha mostrato, in confronto alla ciclofosfamide, un'efficacia pari o addirittura superiore nell'indurre la remissione della nefrite lupica severa e minori effetti collaterali, in particolare un ridotto rischio di infezioni e leucopenia; anche nel mantenimento può costituire una valida alternativa all'azatioprina [26].

Nel caso in esame, l'alto titolo di anticorpi antifosfolipidi aumentava la probabilità di eventi tromboembolici e, sebbene non vi sia correlazione fra il titolo anticorpale aPL e i livelli di anticorpi anti-DNA e/o di complemento, l'APSN sovrapposta alla nefrite lupica rappresenta un fattore di rischio aggiuntivo per morbilità renale, ipertensione e fibrosi interstiziale, questi ultimi riconosciuti indicatori prognostici della funzione renale [27].

È probabile che l'APSN partecipi alla progressione verso l'ESRD (End-Stage Renal Disease), così com'è già stato descritto nell'APS in assenza di nefrite lupica [28]. Di qui la necessità di continuare a mantenere un'anticoagulazione più intensiva che in passato con target INR 3-4, in accordo con le raccomandazioni della European League Against Rheumatism (EULAR) del 2008 [29].

L'APS è quindi associata al LES e può precederne la comparsa anche a distanza di molti anni. Nelle serie riportate in letteratura, la comparsa di LES e sindrome lupus-like oscilla fra il $9 \%$ e il $13 \%$ al follow-up di 6 e 8 anni $[30,31]$ e vi sono report a distanza di un decennio o più $[32,33]$. Poiché l'APS può essere la prima manifestazione del lupus, riteniamo sia utile un follow-up superiore a 5 anni per escludere la possibile emergenza del LES [21-34].

\section{Ringraziamenti}

Gli autori ringraziano i colleghi U. Ferbo, A. Ciampa e S. De Silva per il fattivo contributo offerto al caso in esame.

\section{Conflitto di interesse}

Gli autori dichiarano di essere esenti da conflitto di interessi.

\section{Bibliografia}

[1] Cervera R, Asherson RA, Lie JT. Clinicopathologic correlations of the antiphospholipid syndrome. Semin Arthritis Rheum 1995; 24(4):262-72.

[2] Harris EN, Gharavi AE, Loizou S, Derue G, Chan JK, Patel BM, et al. Crossreactivity of antiphospholipid antibodies. J Clin Lab Immunol 1985;16(1):1-6.

[3] Galli M, Comfurius P, Maassen C, Hemker HC, de Baets MH, van Breda-Vriesman PJ, et al. Anticardiolipin antibodies (ACA) directed not to cardiolipin but to a plasma protein cofactor. Lancet 1990;335(8705):1544-7.

[4] Wilson WA, Gharavi AE, Koike T, Lockshin MD, Branch DW, Piette JC, et al. International consensus statement on preliminary classification criteria for definite antiphospholipid syndrome: report of an international workshop. Arthritis Rheum 1999;42(7):1309-11.

[5] Miyakis S, Lockshin MD, Atsumi T, Branch DW, Brey RL, Cervera $\mathrm{R}$, et al. International consensus statement on an update of the classification criteria for definite antiphospholipid syndrome (APS). J Thromb Haemost 2006;4(2):295-306.

[6] Sanna G, Bertolaccini ML, Cuadrado MJ, Khamashta MA, Hughes GR. Central nervous system involvement in the antiphospholipid (Hughes) syndrome. Rheumatology (Oxford) 2003; 42(2):200-13.

[7] Meroni PL, Tincani A, Sepp N, Raschi E, Testoni C, Corsini E, et al. Endothelium and the brain in CNS lupus. Lupus 2003;12(12):919-28.

[8] Sangle S, D'Cruz D, Khamashta M, Hughes GRV. Prevalence of hypertension in 600 patients with antiphospholipid syndrome. Rheumatology (Oxford) 2004;43(Suppl 2):S105-9.

[9] Nochy D, Barres D, Camilleri JP, Bariety J, Corvol P, Menard J. Abnormalities of renin-containing cells in human glomerular and vascular renal diseases. Kidney Int 1983;23(2):375-9.

[10] Nochy D, Daugas E, Droz D, Beaufils H, Grünfeld JP, Piette JC, et al. The intrarenal vascular lesions associated with primary antiphospholipid syndrome. J Am Soc Nephrol 1999;10(3): 507-18.

[11] Becquemont L, Thervet E, Rondeau E, Lacave R, Mougenot B, Sraer JD. Systemic and renal fibrinolytic activity in a patient with anticardiolipin syndrome and renal thrombotic microangiopathy. Am J Nephrol 1990;10(3):254-8.

[12] Daugas E, Nochy D, Huong DL, Duhaut P, Beaufils H, Caudwell V, et al. Antiphospholipid syndrome nephropathy in systemic lupus erythematosus. J Am Soc Nephrol 2002;13(1):42-52.

[13] D’Agati V, Kunis C, Williams G, Appel GB. Anti-cardiolipin antibody and renal disease: a report three cases. J Am Soc Nephrol 1990;1(5):777-84.

[14] Dorel M, Daniel L, Liprandi A, Lerda D, Pellissier JF. Idiopathic membranous glomerulonephritis associated with primary antiphospholipid syndrome. Nephron 2000;86(3):366-7.

[15] Fakhouri F, Noël LH, Zuber J, Beaufils H, Martinez F, Lebon P, et al. The expanding spectrum of renal diseases associated with antiphospholipid syndrome. Am J Kidney Dis 2003;41(6): 1205-11.

[16] Manganelli R, lannaccone $S$, lannaccone $M$, De Simone E, Ferbo U, Nappi F, et al. Diagnostic pathway in a case with severe degree of hypertension. G Ital Nefrol 2006;23(4): $442-6$.

[17] Cervera R, Piette JC, Font J, Khamashta MA, Shoenfeld Y, Camps MT, et al., Euro-Phospholipid Project Group. Antiphospholipid syndrome: clinical and immunologic manifestations and patterns of disease expression in a cohort of 1,000 patients. Arthritis Rheum 2002;46(4):1019-27.

[18] Hochberg MC. Updating the American College of Rheumatology revised criteria for the classification of systemic lupus erythematosus. Arthritis Rheum 1997;40(9):1725. 
[19] Tincani A. Antiphospholipid antibodies and other autoantibodies. In: Proceedings of $5^{\text {th }}$ International Symposium on Antiphospholipid Antibodies; 1992.

[20] Vianna JL, Khamashta MA, Ordi-Ros J, Font J, Cervera R, LopezSoto A, et al. Comparison of the primary and secondary antiphospholipid syndrome: a European multicenter study of 114 patients. Am J Med 1994;96(1):3-9.

[21] Piette JC, Wechsler B, Francis C, Godeau P. Systemic lupus erythematosus and the antiphospholipid syndrome: reflections about the relevance of ARA criteria. J Rheumatol 1992;19(12): 1835-7.

[22] Cameron JS. Lupus nephritis. J Am Soc Nephrol 1999;10(2): 413-24.

[23] Weening JJ, D’Agati VD, Schwartz MM, Seshan SV, Alpers CE, Appel GB, et al., International Society of Nephrology Working Group on the Classification of Lupus Nephritis. Renal Pathology Society Working Group on the Classification of Lupus Nephritis. The classification of glomerulonephritis in systemic lupus erythematosus revisited. Kidney Int 2004;65(2):521-30.

[24] Kashgarian M. Lupus nephritis: lessons from the path lab. Kidney Int 1994;45(3):928-38.

[25] Grande JP, Balow JE. Renal biopsy in lupus nephritis. Lupus 1998;7(9):611-7.

[26] Zhu B, Chen N, Lin Y, Ren H, Zhang W, Wang W, et al. Mycophenolate mofetil in induction and maintenance therapy of severe lupus nephritis: a meta-analysis of randomized controlled trials. Nephrol Dial Transplant 2007;22(7):1933-42.

[27] Klahr S. Mechanisms of progression of chronic renal damage. J Nephrol 1999;12(Suppl 2):S53-62.
[28] Dayal NA, Isenberg DA. Endstage renal failure in primary antiphospholipid syndrome - case report and review of literature. Rheumatology (Oxford) 2003;42(9):1128-9.

[29] Bertsias G, loannidis JP, Boletis J, Bombardieri S, Cervera R, Dostal C, et al., Task Force of the EULAR Standing Committee for International Clinical Studies Including Therapeutics. EULAR recommendations for the management of systemic lupus erythematosus. Report of a Task Force of the EULAR Standing Committee for International Clinical Studies Including Therapeutics. Ann Rheum Dis 2008;67(2):195-205.

[30] Carbone J, Orera M, Rodríguez-Mahou M, Rodríguez-Pérez C, Sánchez-Ramón S, Seoane E, et al. Immunological abnormalities in primary APS evolving into SLE: 6 years follow-up in women with repeated pregnancy loss. Lupus 1999;8(4):274-8.

[31] Gómez-Puerta JA, Martín H, Amigo MC, Aguirre MA, Camps MT, Cuadrado MJ, et al. Long-term follow-up in 128 patients with primary antiphospholipid syndrome: do they develop lupus? Medicine (Baltimore) 2005;84(4):225-30.

[32] Al Attia HM. Progression of primary APS (Hughes syndrome) into serological SLE: case report. Rheumatol Int 2001;20(2):79-80.

[33] D’Agati VD. Renal disease in systemic lupus erythematosus, mixed connective tissue disease, Sjøgren's syndrome, and rheumatoid arthritis. In: Jennette JC, Olson IL, Schwartz MM, Silva FG, editors. Heptinstall's Pathology of the Kidney. 6th Ed., Philadelphia (PA): Lippincott Williams \& Wilkins; 2007 . p. 517-612.

[34] Asherson RA, Baguley E, Pal C, Hughes GR. Antiphospholipid syndrome: five year follow up. Ann Rheum Dis 1991;50(11): 805-10. 